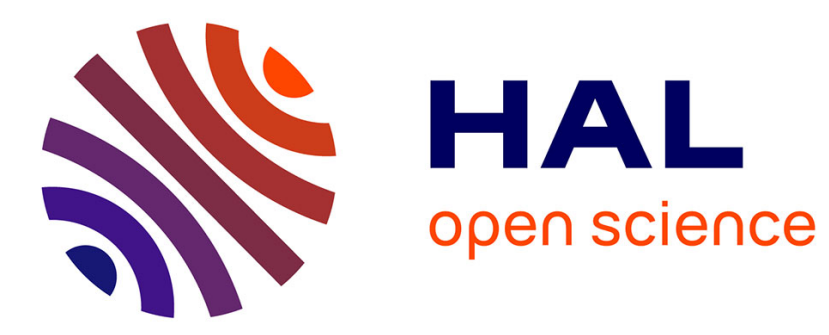

\title{
Evolutionary supervision of a dynamical neural network allows learning with on-going weights
}

David Meunier, Hélène Paugam-Moisy

\section{To cite this version:}

David Meunier, Hélène Paugam-Moisy. Evolutionary supervision of a dynamical neural network allows learning with on-going weights. 2005, pp.1493-1498. hal-00008702

HAL Id: hal-00008702

https://hal.science/hal-00008702

Submitted on 13 Sep 2005

HAL is a multi-disciplinary open access archive for the deposit and dissemination of scientific research documents, whether they are published or not. The documents may come from teaching and research institutions in France or abroad, or from public or private research centers.
L'archive ouverte pluridisciplinaire HAL, est destinée au dépôt et à la diffusion de documents scientifiques de niveau recherche, publiés ou non, émanant des établissements d'enseignement et de recherche français ou étrangers, des laboratoires publics ou privés. 


\title{
Evolutionary supervision of a dynamical neural network allows learning with on-going weights
}

\author{
September 13, 2005
}

David Meunier and Hélène Paugam-Moisy

Institut des Sciences Cognitives, UMR CNRS 5015

67, boulevard Pinel F-69675 Bron cedex - France

E-mail: \{dmeunier,hpaugam $\} @$ isc.cnrs.fr

Published in:

Proceedings of International Joint Conference on Neural Networks (IJCNN) 2005

pp. $1493-1498$

\begin{abstract}
Recent electrophysiological data show that synaptic weights are highly influenced by electrical activities displayed by neurons. Weights are not stable as assumed in classical neural network models. What is the nature of engrams, if not stored in synaptic weights? Adopting the theory of dynamical systems, which allows an implicit form of memory, we propose a new framework for learning, where synaptic weights are continuously adapted. Evolutionary computation has been applied to a population of dynamic neural networks evolving in a prey-predator environment. Each individual develops complex dynamic patterns of neuronal activity, underlied by multiple recurrent connections. We show that this method allows the emergence of learning capability through generations, as a by-product of evolution, since the behavioural performance of the network is not a priori based on this property.
\end{abstract}

\section{Introduction}

Brain components are quite more dynamical than assumed up to now in connectionist models, both at synaptic and neuronal levels. The "Spike-Timing Hypothesis", or "Temporal Correlation Hypothesis" [48, 47, 19] assumes that each action potential conveys pertinent information [44]. Electrophysiological experiments have shown that the synchronisation among groups of neurons is stimulus-dependent $[20,12]$ and that the causal order between times of emission (pre-to-post) highly influences the synaptic strength $[36,6]$.

However, from a connectionist point of view, it raises the question of the nature of engrams [32, 23]. It is widely accepted that synaptic weights are the support of learning, computed in a preliminary step (learning phase), and kept fixed during the whole 
computation (generalisation phase) of the network. If synaptic weights do not stop varying, what is the nature of the storage of "souvenirs"? This problem has long been described as stability/plasticity dilemna [7]. When confronted to continuous sequences of inputs, such a system will face "catastrophic forgetting" $[4,14]$, that is the forgetting of old patterns due to the presentation of new ones.

The theory of dynamical systems offers another point of view. In dynamical systems, a given state depends on all previously encountered situations, and thus allows a form of memory [10]. Several authors have suggested that this theory [46, 33], and even chaotic dynamical systems $[42,13,45,27,28]$ could be used to describe the electrical activity of the brain. Due to the multiple retroactions between neurons (or "circuits", [43]), non-linearity could emerge at the network level rather than inside the model of neuron [25, 27, 31]. Multiple retroactions are assumed to mimic "poly-synaptic" loops [34], which have been described as being responsible for long time-scale cognitive properties [11]. However, by nature, a dynamical system is very hard to regulate. In real-world environments, natural selection is the way to increase the efficiency of individuals. We propose to develop a virtual environment for selecting the best dynamical systems (recurrent neural networks) by means of an external criterion which is based on the resultant behaviour of individuals "equipped" with such networks [21]. Evolution allows to supervise the way the networks work, whatever might be their complexity [29]. Afterwards, we can analyse, in terms of learning capacity, the improvement of the networks resulting from the evolutionary process.

We have assumed an intrinsic temporal behaviour for synapse and neuron models, by coding precise times of emission for the generation of action potentials. We control their impact on synapses through comparison of pre-synaptic and post-synaptic times of emission, using STDP ("Spike Time Dependent Plasticity"). At the beginning, the topology of the networks is set arbitrarily. Along the evolutionary process, the best topologies are selected according to the performance of an individual, moving in a prey-predator environment, with the network as "brain". This allows to have an objective evaluation based on the resultant behaviour of the individual, whatever might be the internal dynamics of its network. The parameters whithin the neuron and synapse models are fixed all along the evolution, and rather than studying the properties of the networks with a given set of parameters, evolutionary computation allows to study the emergence of topologies based on a given set of values. We would like to emphasize here that topologies are not based on the imitation of a given biological neural network. They involve multiple inhibitory and excitatory connections, and biologyinspired synaptic plasticity. The article shows that the resultant individuals are more and more capable of retaining associations between perceptive inputs and motor outputs, although the network displays complex patterns of activity [38] and the weights do not stop varying "life-long".

\section{Materials \& Methods}

\subsection{Network Model}

\subsubsection{Neuron Model}

Spike Response Model [17, 16] assumes that the neuron state depends only on the last time of firing of the neuron, noted $t_{i}^{f}$. Here we use simplified form for the PSP kernel, namely a dirac $\delta(t)$ to improve computing costs, but retaining a fondamental mode of 
computing of biological neurons, synchrony detection [2, 30, 35]. Figure 1 displays the computing of a single neuron. Membrane potential consists of a sum of incoming post-synaptic potentials (PSPs). The impact of an excitatory PSP is instantaneous, the membrane potential being updated proportionnaly to the weight of PSP impact, with a maximum impact of $2 \mathrm{mV}$.

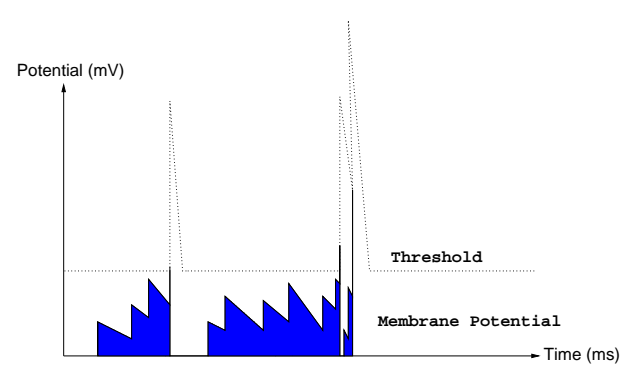

Figure 1: Example of the impact of excitatory PSPs on the neuron membrane and threshold potential

Inhibitory PSPs act in the same way, with a maximal value of $-2 \mathrm{mV}$, if membrane potential is not under a minimal hyperpolarisation potential $(-20 \mathrm{mV})$. The membrane potential is updated between two impacts through an exponential decrease of time constant $\tau_{m e m}=20 \mathrm{~ms}$. For neuron $\mathrm{j}$,

$$
\frac{d u_{j}(t)}{d t}=-\tau_{m e m} u_{j}(t)+\sum_{i=1}^{n} 2 \omega_{i j} \delta\left(t-t_{i}^{f}-d_{i j}\right)
$$

where $n$ is the number of PSPs at time $t, \omega_{i j}$ is the weight and $d_{i j}$ is the delay of synapse between neurons $i$ and $j$. A spike emission occurs when membrane potential is greater than threshold potential, which is initially set to a reference value $\left(\theta_{\text {ref }}=20 \mathrm{mV}\right)$. The membrane potential is reset to resting potential. The threshold potential is added a high value $\left(\theta_{\max }=2 \times \theta_{\text {ref }}\right.$, modelling absolute refractory period $\left(\tau_{a b s}=10 \mathrm{~ms}\right)$, and then goes back to $\theta_{\text {ref }}$, modelling a relative refractory period $\left(\tau_{r e l}=10 \mathrm{~ms}\right)$.

$$
u_{j}(t) \geqslant \theta_{j}(t) \Longrightarrow\left\{\begin{aligned}
u_{j}(t) & \leftarrow u_{\text {rest }} \\
\theta_{j}(t) & \leftarrow \theta_{j}(t)+\theta_{\max } \\
t_{j}^{(f)} & \leftarrow t
\end{aligned}\right.
$$

Between two spikes, the threshold potential is updated according to equation (3), allowing to model the refractory period:

$$
\frac{d \theta_{j}(t)}{d t}= \begin{cases}-\tau_{\text {rel }}\left(\theta_{j}(t)-\theta_{\text {ref }}\right) & \text { if } t-t_{j}^{(f)}>\tau_{a b s} \\ 0 & \text { otherwise }\end{cases}
$$

This way of computing the threshold allows the neuron to burst (right part of figure 1).

\subsubsection{Synapse Model}

Spike-Time Dependent Plasticity (STDP) involves a kind of synaptic plasticity, based on the difference between times of pre- and post-synaptic firing [6]. If the times of emission follow a causal order (pre-to-post), the weight is potentiated, and deprecated 
otherwise. Each synapse has access locally to the times of post-synaptic neuron firings via a back-propagated spike [36]. Such a modelling of biological data implies that the weight of the synapse is updated at each time the neuron (either pre- or post-synaptic) emits a spike [39][26]. The classical version of STDP $[15,1,16]$ is based on delay selection, and involves a discontinuity between potentiation and depression zones of temporal window : the synapse emitting a PSP that will be responsible for the spike emission is the most potentiated, whereas a PSP arriving just after a spike will be the most deprecated. However, the electrophysiological data shown in [6] can also be modelled with a very fast rising potentiation zone (figure 2).
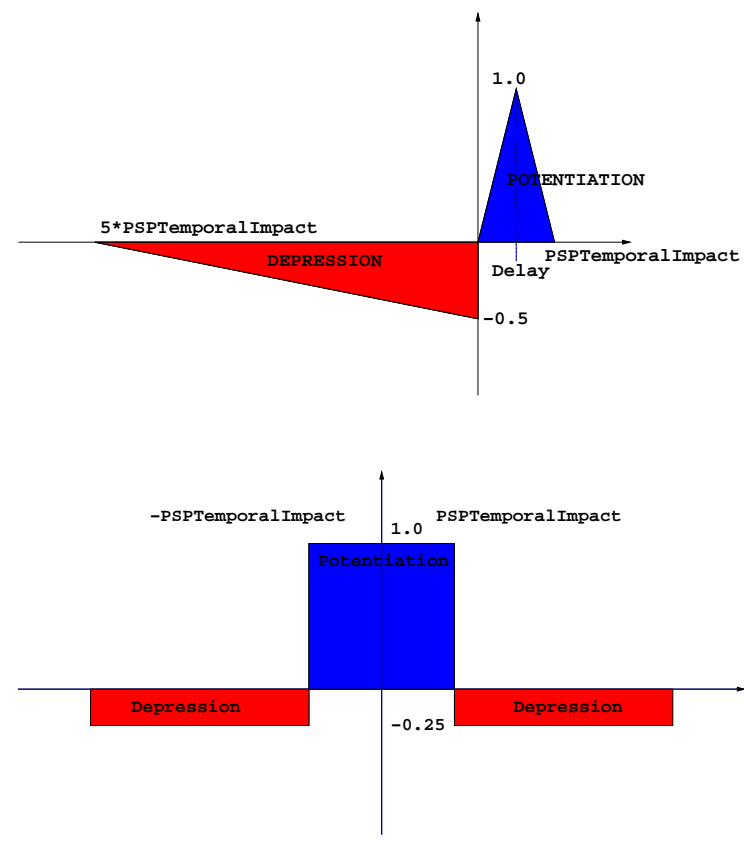

Figure 2: Assymetric temporal window used for excitatory synapses (up, based on [6]) and inhibitory synapses (down, based on [49]

The window is defined to match the delays of the synapse, "Delay" on figure 2 corresponding to the maximum delay $(10 \mathrm{~ms})$, whereas "PSPTemporalImpact" refers to the time constant of membrane potential decay, $\tau_{m e m}$ (20ms). In [39], Nowotny et al. describe a model of temporal window based on a regulation process, via the cumulated effects of two successive applications of the temporal window. For each positive difference of $t_{\text {post }}-t_{\text {pre }}$, there exists an homologous negative value of $t_{\text {post }}-t_{\text {pre }}$, so that the successive application of the temporal window corresponding to these two values leave the weight unchanged. The process described in [39] shows that, through time, the weight tends to a value that allows the adequation with the pre-synaptic and postsynaptic spike trains. After having tried the classical version of STDP, we have used a linear version of the assymetric window described in [39], because this regulation process is well adapted to our model involving multiple retroactions.

Inhibitory synapses are updated according to a quite different temporal window (figure 2), based on correlation of spikes, and where the order has no influence [49]. In this case, inhibition plays a role of regulation rather than "shunting" the post-synaptic 
neuron activity.

Each weight is initialised with the value 0.5 for excitatory synapses ( -0.5 for inhibitory synapses), and each delay is chosen randomly between 1 and $10 \mathrm{~ms}$ for each synapse, but kept fixed during the rest of the simulation. Both inhibitory and excitatory synapse models are updated by adding the result of the application of the temporal window to the current value, ponderated by an $\alpha$ value (0.1), and by a smoothing mechanism so that the weight stays in the range $[0,1]$ for excitatory synapses (or $[-1,0]$ for inhibitory synapses).

\subsubsection{Network Architecture}

Assemblies (groups of neurons) are linked by projections, i.e. all-to-all synaptic links between the neurons of the assemblies. A projection is either excitatory or inhibitory, and is unidirectional. Assemblies either correspond to input assemblies (left on figure 3 ), internal assemblies (center) or output assemblies (right). Input assemblies can only send projections towards other assemblies, output assemblies can only receive projections from assemblies. Internal assemblies are the only ones to both receive and send projections. We have chosen the number of projections so that each internal assembly receives in average 6 input projection, and sends 6 output projections.

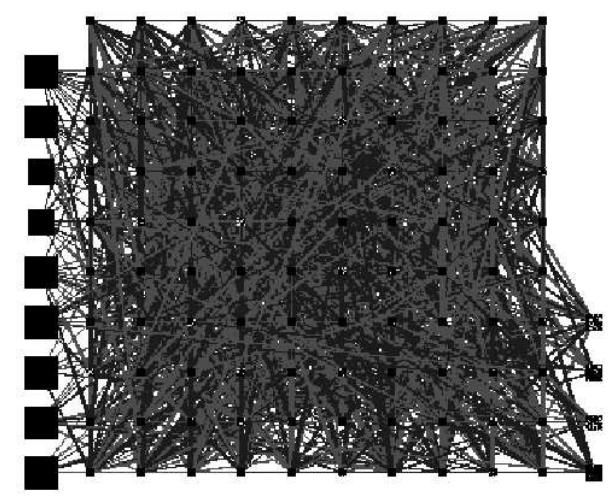

Figure 3: Network topology involving 100 assemblies of 25 internal neurons each, 9 input assemblies (5 vision - 2 audition - 1 punish - 1 reward) and 4 motor output assemblies ( 2 body -2 head)

\subsection{Environment Model}

\subsubsection{Prey-Predator Environment}

The experimental platform is a virtual environment (a "virtual zoo", previously developed in [40], [8]) where a virtual robot (upper left corner on figure 4), equipped with a neural network as "brain", moves among animals. Usually, ecologists are interested in the evolution of demography in prey-predator dynamics, whereas computer scientists may study the mechanisms of evolving communicating agents for the prey-predator pursuit problem. Our purpose is different and more cognitive. We aim at optimising the life span of an individual (a "virtual robot") evolving among a world inhabited by 
animals which can be preys (in black), predators (dark grey) or neutral animals (light grey) w.r.t. it.

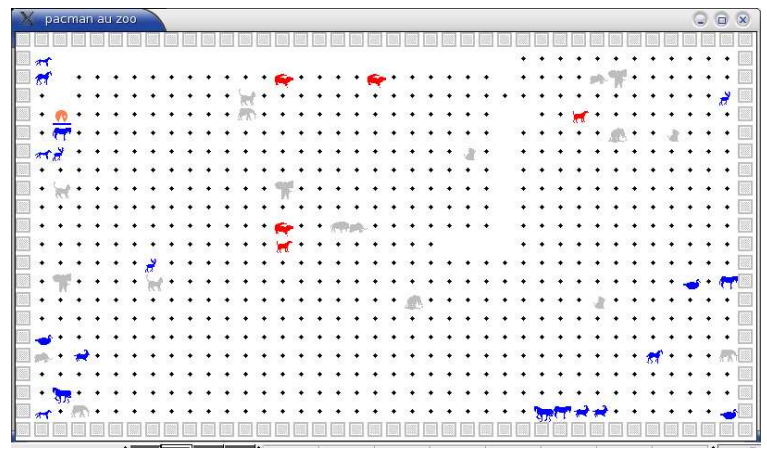

Figure 4: Virtual environnement involving an individual with preys (in blue), predators (in red) and neutral animals (in grey)

\subsubsection{Structure of a Virtual Robot}

The robot perceived the virtual zoo via 2 modalities (vision, audition). Visual patterns correspond to images of $20 * 20$ pixels representing animals, auditory patterns are the discretized spectrum of frequencies corresponding to the sound emitted by real corresponding animals [40]. Vision consists of 5 assemblies of 400 neurons, each assembly corresponds to a column in the zoo, starting from the robot, in the direction of its "head", indicated with a black bar on figure 4. The first animal present in a column is sent to the corresponding assembly. Audition consists of 2 assemblies of 256 neurons, receiving inputs if an animal is in a restricted zone of $5 * 5$ around the robot. If the animal is on the left (resp. right) of the head, only the left (resp. right) ear receives inputs. If the animal is in front or behind, both ears receive inputs. Patterns of both modalities are noised according to the distance between the animal and the robot. Another modality, corresponding to conditioning inputs, informs the network of either a predator contact (punish input), or of the eating of a prey or of a gum (reward input). Injection in the network is achieved via a stochastic process, each value being normalized between 0.0 and 1.0 forcing the emission of a spike by corresponding input neurons each with a probability of $10 *$ value\% each time step.

Motor outputs are controlled by 4 assemblies of neurons: 2 for the head and 2 for the body. Each motor output determines a direction (turning body, resp. turning head), and if the two assemblies are activated together, the robot moves ahead (resp. put head in the same direction as the body).

\subsection{Evolutionary Model}

\subsubsection{Network Topology Encoding}

We have based the design of the network topology on a modified version of a classical encoding, known as projection encoding, or layer-based encoding [22]. Each projection is coded by of three integer genes, the first one corresponding to the source assembly index, the second consists in a projection code, describing the excitatory or inhibitory nature of the synapses ( 0 or 1$)$, and the third corresponds to the target assembly index. 
Since the network is composed of 100 assemblies of 25 internal neurons, plus 9 input and 4 output assemblies, the total number of projections is 1278 . Hence a chromosome is composed of 3834 genes. Finally, a typical network have 6212 neurons, and $\sim 2000000$ synapses, depending on the number of internal-hidden and internal-output projections.

\subsubsection{Fitness Function}

At each run, the robot starts with an initial "life credit" (200 points), and the fitness function is computed by accumulating credit modifications at each time step. The life level is increased when the robot eats either "gums" (small black dots in figure $4,+20$ points) or preys (+200 points). Predators "hurt" the robot when in contact with it $(-2$ points each time step), and the life level is decreased each fifth time step (-1 point).

Hence the fitness fonction measures the three fondamental properties that we want to see emerging with evolution:

- Zoo exploration, via the number of eaten gums.

- Prey approaching, via the number of eaten preys.

- Predator avoidance is implicitly involved in the function since the time during which the robot survives depends on the number of life credit accumulations.

\subsubsection{Evolutionary Algorithm}

The evolutionary algorithm is a classical version of original genetic algorithms [18] with the integer encoding described above. For the three main phases of evolution, we used:

- Evaluation is realised by building the network corresponding to a chromosome, by equipping the virtual robot with the resultant network, and letting the robot in the virtual environment. The fitness of an individual is the lowest value of 2 evaluations, allowing to select primarily those individual whose performance is reproducible.

- Selection is achieved by tournaments. This consists in defining the size of each tournament, to select randomly individuals from the population, and to make copy of the best individual within the tournament to the next population. Classically, the tournament size should be half or third the total population size.

- Variation includes the two classical genetic operators, i.e. mutation and crossingover, with rate chosen so that there is in average one mutation on each chromosome and one crossing-over each two chromosomes.

\subsection{Learning test}

\subsubsection{Test Lab}

We have realized "behavioural" tests to measure correspondances between the visual and auditory patterns for a given animal, and the adapted behaviour (avoidance for predators, approach for preys). We have defined a smaller environment, of size $5 * 8$ (the "test lab"), where the robot and one other animal, prey or predator, are always located at the same place, the individual initially always faces the animal. The animal 
does not move, and the robot is allowed to move during a fixed amount of time. The score of the robot is evaluated with its behaviour:

- if the animal is a prey, a good behaviour will be to catch the animal, a bad behaviour will be to avoid the animal (i.e. escaping out of the test lab).

- if the animal is a predator, a good behaviour will be to avoid the animal (i.e. escaping out of the test lab), a bad behaviour will be to catch the animal.

Whenever the robot has neither escaped the test lab, nor caught the animal, we state in all cases a bad behaviour.

\subsubsection{Test Protocol}

The network is tested at two moments, one time before the run in the zoo, and the other after the run. The first test score allows to measure "innate" knowledge of the individual about the environment. The difference between the second and the first tests is a measure of how the individual has learned "during its life", after having met animals. Notice that conditioning inputs are activated all along the runs, but never during the behavioural tests.

\section{Results}

We show results for an evolutionary run of 30 individuals, during 15 generations. Figure 5 (up) displays the performance for all the individuals. Each generation is represented by a single line, where the individuals are sorted according to their fitness. Best individuals in each genration are highlighted.

Figure 5 (down) shows the mean fitness for each generation. Since the performance is evaluated through the behaviour of the individuals in the zoo, the figure shows that the evolutionary algorithm allows to build more and more adapted individuals.

Figure 6 shows the mean score in the test lab for every other generation. Each individual is tested in 100 test labs, involving randomly chosen animals in the zoo, but alternating preys and predators. The test score is the difference between good behaviour and bad behaviour scores. The red line represents the score before the run in the zoo, the green line after the run in the zoo. The initial (random) individuals display no particular ability in their behaviour, and no particular ability to learn. It can be seen that the individuals gain "innate" knowledge about the environment, corresponding to the test before the run. Moreover, they gain more and more capacity to learn after having met the animals in the zoo.

To illustrate the fact that our learning rule is not based on synaptic weights fixation, figure 7 displays an example of a neuron (up) and all its associated synapses during the run of an individual. It can be seen that synapses do not stop moving, at each time the neuron emits a spike. However, synapses display different variations, depending on the spike trains of the other connected neuron.

\section{Discussion}

Up to now, nobody (to the best of our knowledge) has been able to show how it is possible to learn with STDP, although there are growing evidence that such a mechanism is 

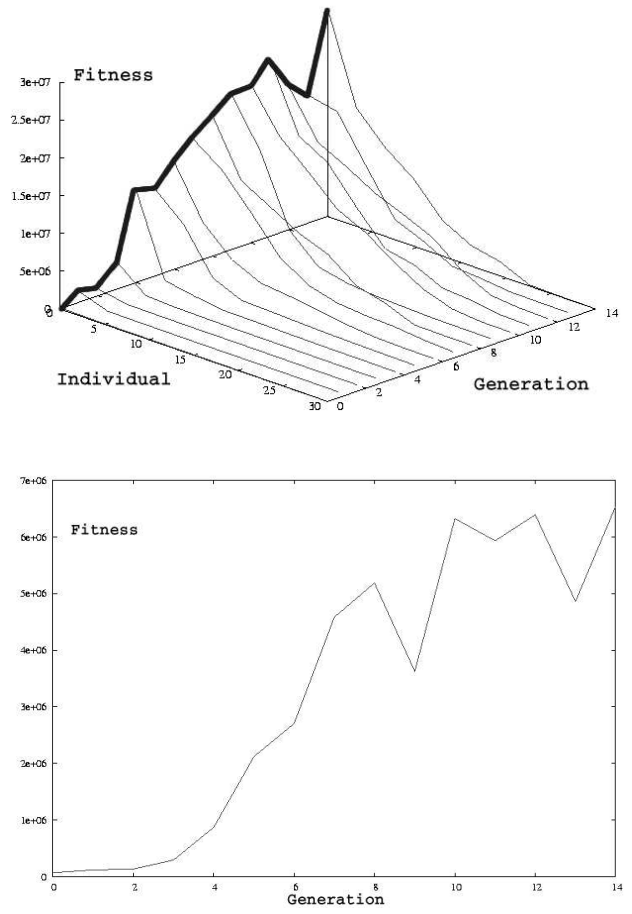

Figure 5: Performance of the sorted individuals for all generations (up). Best individuals of each generation are highlighted. Mean performance for the individuals of a generation (down), for all generations

quite general in the nervous system [9]. We have used an ecologically plausible mechanism to show that evolution is able to select the individuals whose mapping between modal inputs and motor outputs produce adapted behaviour. The learning test allows the evaluation of how well the network is able to associate punish/reward signals with the modal patterns corresponding to animals. However, our protocole also includes the ability to move, either to approach preys, or to escape predators.

Figure 6 shows that initially, evolution favours the individuals that are able to move in the zoo, involving a quite better score in the test before run (generation 2-4). When most of the individuals are able to move, evolution favours those that are able to correctly adapt their behaviour to the situation, using conditioning inputs (generation 1014). As evolution is a differential mechanism, it allows an incremental increase in the performance of the individual. The results on learning properties are here considered as by-product of evolution: Although the performance of the individuals do not explicitely depend on their ability to learn, we have shown that the test score after run is better than the score before run. It should be noticed that, as the zoo is the only place where the individuals have access to information about the nature prey/predator via conditioning inputs, the fact of living quite a longer time allows the individuals to face many more animals, and hence to be more efficient in the second run.

The effect observed here is quite close to the so-called "Baldwin effect" [5], that is the effect of learning on natural selection. Although originately described as a Lamarckian process (heritability of acquired character), it has been shown that the Darwinian theory could also explain this effect, by considering the benefits of learning to survive, 


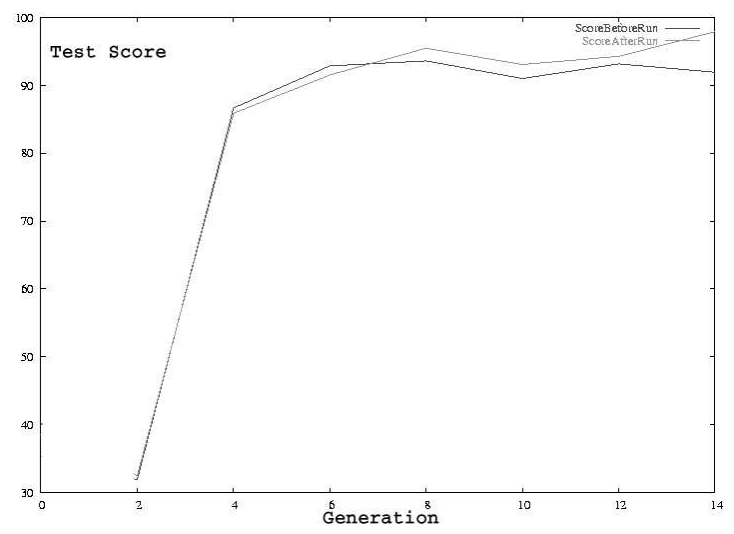

Figure 6: Mean score for each 2 generations, each individual being tested 100 times. Tests before (red) and after (green) the run in the zoo

and thus act on natural selection [41]. There has been several models of involving Baldwin effect [24, 3, 37], but all of them explicitely encode innate knowledge and learned knowledge with the same representation, i.e. synaptic weights. Here we provide a model with no explicit representation of innate knowledge, but where this effect appears however.

\section{Conclusion}

We have presented a method, based on evolutionary algorithms, for building individuals that are adapted to a given environment. Neural networks are composed of spiking neurons and dynamic synapses, and their non-linearity is due to their highly recurrent topology, involving multiple retroactions. We have been able to show that, using an innovative framework based on the dynamical hypothesis for the nature of engrams, the networks are able to learn correlations between percepts and behaviours, although the memory is not based on static weights.

\section{References}

[1] L. F. Abbott and S. B. Nelson. Synaptic plastictiy - Taming the beast. Nature Neurosci., 3:1178-1183, 2000.

[2] M. Abeles. Corticonics. Cambridge Univ. Press, 1991.

[3] L. W. Ancel. A quantitative model of the simpson-baldwin effect. Journal of Theoretical Biology, 196:197-209, 1999.

[4] B. Ans and S. Rousset. Neural networks with a self-refreshing memory: Knowledge transfer in sequential learning tasks without catastrophic forgetting. Connection science, 12:1-19, 2000.

[5] J. M. Baldwin. A new factor in evolution. American Naturalist, 30:441-451, 1896. 

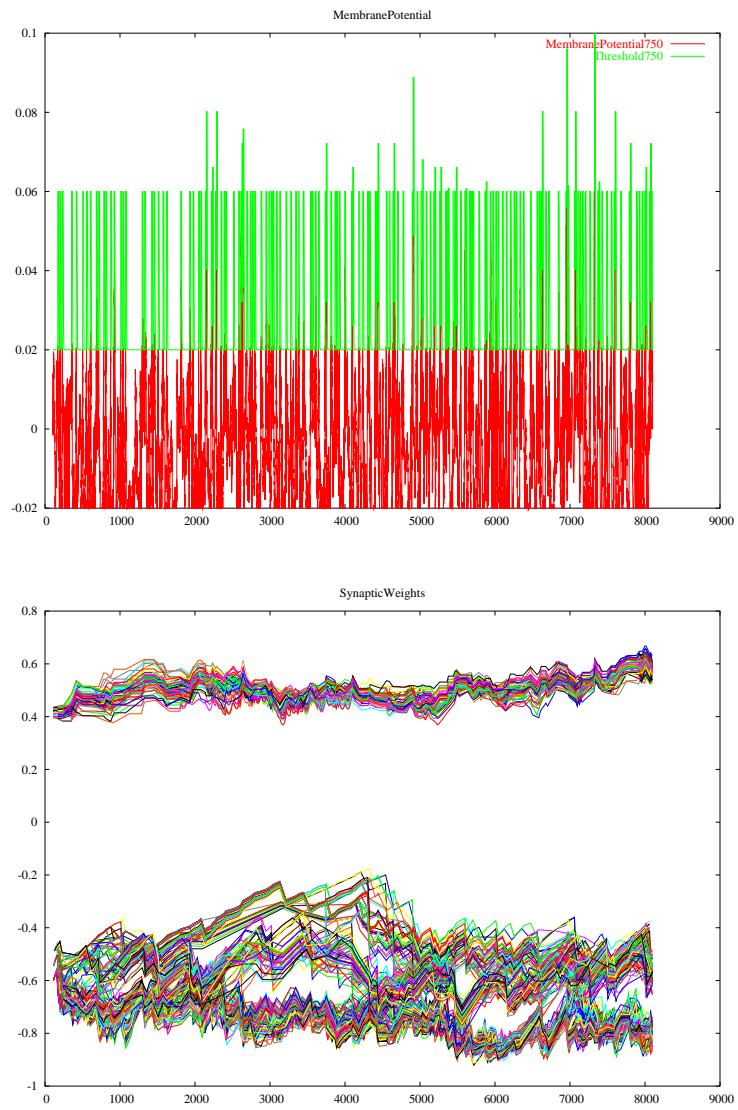

Figure 7: Membrane potential and threshold of a neuron (up), and the weights of its associated input and output synapses (down) during a run in the zoo

[6] G. Bi and M. Poo. Synaptic modifications in cultured hippocampal neurons: dependence on spike timing, synaptic strength, and postsynaptic cell type. $J$. Neurosci., 18:10464-10472, 1998.

[7] G.A. Carpenter and S. Grossberg. Pattern Recognition by Self-Organizing Neural Networks. MIT Press, Cambridge, Mass., 1991.

[8] S. Chevallier, H. Paugam-Moisy, and F. Lemaitre. Distributed processing for modelling real-time multimodal perception in a virtual robot. In International Conference on Parallel and Distributed Computing and Networks. IASTED, ACTA press (to appear), 2005.

[9] Y. Dan and MM. Poo. Spike timing-dependent plasticity of neural circuits. Neuron, 44(1):23-30.

[10] J. Demongeot, M. Kauffman, and R. Thomas. Positive feedack circuits and memory. Comptes-rendu de l'Acadmie des Sciences de Paris, Sciences de la vie, 323:69-79, 2000.

[11] G. M. Edelman. Neural Darwinism: the theory of neuronal group selection. Basic Books, New York, 1987. 
[12] A. K. Engel, P. Konig, and W. Singer. Direct physiological evidence for scene segmentation by temporal coding. Proc. Nat. Ac. Sci., 88:9136-9140, 1991.

[13] W. J. Freeman. Role of chaotic dynamics in neural plasticity, 1994.

[14] R. M. French. Catastrophic forgetting in connectionist networks. Trends in Cognitive Sciences, 3, 1999.

[15] W. Gerstner, R. Kempter, J.L. van Hemmen, and H. Wagner. A neuronal learning rule for sub-millisecond temporal coding. Nature, 383:76-78, 1996.

[16] W. Gerstner and W. Kistler. Spiking Neuron Models: Single Neurons, Populations, Plasticity. Cambridge University Press, 2002.

[17] W. Gerstner, R. Ritz, and J.L. van Hemmen. Why spikes? hebbian learning and retrieval of time-resolved excitation patterns. Biol. Cybern., 69:503-515, 1993.

[18] D. E. Goldberg. Genetic algorithms in search, optimisation and machine learning. Addison-Wesley, Reading MA, 1989.

[19] C. M. Gray. The temporal correlation hypothesis of visual feature integration: still alive and well. Neuron, 24, 1999.

[20] C. M. Gray and W. Singer. Stimulus specific neuronal oscillations in orientation columns of cat visual cortex. Proc. Natl. Acad. Sci., 86:1698-1702, 1989.

[21] A. Guillot and E. Dauce. Approche dynamique de la cognition artificielle, chapter Conclusion. Lavoisier, Paris, 2002.

[22] S.A. Harp, T. Samad, and A. Guha. Towards the genetic synthesis of neural networks. In Proceedings of the Third International Conference on Genetic Algorithms, pages 360-369, George Mason University, 1989.

[23] D. O. Hebb. The organisation of behaviour: a neurolophysiological theory. Wiley, New York, 1949.

[24] G.E. Hinton and S.J. Nowlan. How learning guide evolution. Complex Systems, 1:495-502, 1987.

[25] J. Ito and K. Kaneko. Self-organized hierarchical structure in a plastic network of chaotic units. Neural Networks, 13, 2000.

[26] Eugene M. Izhikevich, Joseph A. Gally, and Gerald M. Edelman. Spike-timing dynamics of neuronal groups. Cerebral Cortex, 14(8):933-944, 2004.

[27] K. Kaneko and I. Tsuda. Chaotic itinerancy. Chaos, 13(3):926-936, 2003.

[28] L. M. Kay. A challenge to chaotic itinerancy from brain dynamics. Chaos, 13(3):1057-1066, 2003.

[29] C. Koch and G. Laurent. Complexity and the nervous system. Science, 284:9698, 1999.

[30] P. König, A. K. Engel, and W. Singer. Integrator or coincidence detector? the role of the cortical neuron revisited. Trends Neurosci., 19(4):130-137, 1996. 
[31] R. Kozma, W. J. Freeman, and P. Erdi. The KIV model - nonlinear spatiotemporal dynamics of the vertebrate forebrain. Neurocomputing, 52-54:819-826, 2002.

[32] K. S. Lashley. In search of the engram. In J.F. Danielli and R. Brown, editors, Symposia of the Society for Experimental Biology, 4 - Physiological Mechanisms in Animal Behaviour, Cambridge, 1950. Cambridge University Press. Reprinted 1967.

[33] G. Laurent, M. Stopfer, R. Friedrich, M.I. Rabinovich, A. Volkovskii, and H.D.I. Abarbanel. Odor encoding as an active, dynamical process: Experiments, computation and theory. Annu. Rev. Neurosci., 24:263-297, 2001.

[34] E. D. Lumer, G. M. Edelman, and G. Tononi. Neural dynamics in a model of the thalamocortical system i. layers, loops and the emergence of fast synchronous rhythms. Cerebral Cortex, 7:207-227, 1997.

[35] W. Maass. Networks of spiking neurons: the third generation of neural network models. Neural Networks, 10:1659-1671, 1997.

[36] H. Markram, J. Lubke, M. Frotscher, and B. Sakmann. Regulation of synaptic efficacy by coindence of postsynaptic APs and EPSPs. Science, 275:213-215, 1997.

[37] G. Mayley. Guiding or hiding: Explorations into the effects of learning on the rate of evolution. In Phil Husbands and Inman Harvey, editors, Fourth European Conference on Artificial Life, Cambridge, MA, 1997. MIT Press.

[38] D. Meunier and H. Paugam-Moisy. Coherence in a dynamical neural network, and decoherence via modal inputs. in preparation, 2005.

[39] T. Nowotny, V. P. Zigulin, A. I. Selverston, H. D. I. Abarbannel, and M. I. Rabinovitch. Enhancement of synchronization in a hybrid neural circuit by spiketiming dependant plasticity. J. Neurosci., 23(30):9776-9785, 2003.

[40] E. Reynaud and D. Puzenat. A multisensory indentification system for robotics. In IJCNN 2001, pages 2924-2929. International Joint Conference on Neural Networks, 2001.

[41] G. C. Simpson. The baldwin effect. Evolution, 7:110-117, 1953.

[42] C. A. Skarda and W. J. Freeman. How brain makes chaos in order to make sense of the world. Behavioral and Brain Sciences, 10:161-195, 1987.

[43] R. Thomas and M. Kaufman. Multistationnarity, the basis of cell differentiation and memory.I. structural conditions of multistationarity and other nontrivial behavior. Chaos, 11(1):170-179, 2001.

[44] S. J. Thorpe, D. Fize, and C. Marlot. Speed of processing in the human visual system. Nature, 381:520-522, 1996.

[45] I. Tsuda. Toward an interpretation of dynamic neural activity in terms of chaotic dynamical systems. Behavioural and Brain Sciences, 24:793-847, 2001. 
[46] T. J. van Gelder. The dynamical hypothesis in cognitive science. Behavioral and Brain Sciences, 21:1-51, 1998.

[47] C. von der Malsburg. The what and why of binding: The modeler's perspective. Neuron, 24(1):95-104, 1999.

[48] von der Marlsburg. The correlation theory of brain function. Technical report, Max Planck Institute for Biophysical Chemistry, Gttingen, Germany, 1981.

[49] M. Woodin, K. Ganguly, and MM. Poo. Coincident pre- and postsynaptic activity modifies gabaergic synapses by postsynaptic changes in cl- transporter activity. Neuron, 39(5):807-820, 2003. 